\title{
Rudolf Wolf: Lehrer, Forschungsorganisator und Wissenschaftshistoriker. Zu seinem 100. Todesjahr
}

\author{
Beat Glaus
}

SUMMARY ${ }^{1}$

Rudolf Wolf (1816-1893): Astronomer, Research Organizer, Historian of Sciences

Rudolf Wolf is remembered by the scientific community above all as a pioneer of sun spots research. On the centennial anniversary of his death it is appropriate to review his other achievements, which have been wrongly neglected. Wolf organized various astronomical, geodetic and meteorological projects. For example, it was on his initiative that the Federal Observatory in Zurich, designed by the famous architect Gottfried Semper, was built. He taught at academic institutions in Berne and Zurich and developed the library of the Swiss Federal Institute of Technology in Zurich, from its beginnings. His contributions to the history of science are still of interest today: Biographien zur Kulturgeschichte der Schweiz (4 vol., 1858-1862); Geschichte der Astronomie (Munich 1877, reprint 1965); Geschichte der Vermessungen in der Schweiz (1879); Handbuch der Astronomie, ihrer Geschichte und Literatur (2 vol., 1890-1893; reprints 1973).

\section{ZUSAMMENFASSUNG}

Rudolf Wolf, 1816 geboren, studierte an Schulen und Hochschule der Vaterstadt Zürich hauptsächlich Mathematik und Vermessung. 1836-1838 bildete er sich im Ausland weiter, vorzugsweise zu Wien bei Littrow in Astronomie. 1839-1855 war er Mathematiklehrer an der Realschule Bern, ab 1844 Dozent für Mathematik und Astronomie der dortigen Hochschule und seit 1847 Direktor ihrer Sternwarte. Von 1855 bis zu seinem Tode wirkte er erfolgreich als Lehrer, Forschungsorganisator, Fachschriftsteller und -historiker an Eidgenössischer Technischer Hochschule und Universität Zürich. Anerkannt und lexikonwürdig ist Wolf noch als Pionier der Sonnenfleckenforschung. Seine übrigen Leistungen sind nur mehr Spezialisten bekannt; auf sie nimmt der Beitrag deshalb vor allem Bezug. Architekt der Wolf'schen Sternwarte in Zürich war kein Geringerer als Gottfried Semper. 


\section{Quellenlage}

Rudolf Wolfs Leben ist in mehreren, zum Teil umfangreichen Nekrologen recht gut dokumentiert. Fach- und Festschriften der Institutionen, an denen und für die er tätig war, würdigen die einschlägigen Leistungen. Grossen dokumentarischen Wert besitzt das Tagebuch, welches der Jüngling zwischen 1834 und 1841 regelmässig führte ${ }^{2}$, und auch die Biographie des früh verstorbenen Bruders Johann ${ }^{3}$ gibt Einblick ins familiäre und gesellschaftliche Umfeld. Beredtes Zeugnis für Wolfs sozusagen weltweite Verbindungen ist die ab 1841 eingerichtete Autographensammlung der Schweizerischen Naturforschenden Gesellschaft, die sich heute in der Burgerbibliothek Bern befindet. Seltener sind Briefe von Wolfs eigener Hand; darunter ragen die Korrespondenzen mit den lebenslangen Freunden Johann Wild, dem Geodäten (1814-94), und dem Historiker Georg von Wyss (1816-93) hervor. Über seine Vorlesungen an der ETH orientieren lediglich einige eigenhändige Manuskripte und studentische Nachschriften ${ }^{4}$. Ohne allzu grosse Verluste dürften dagegen Wolfs Sammlungen wertvoller Bücher und Instrumente auf uns gekommen sein.

\section{Prägungen}

Die Epoche, in der Wolf aufwuchs, das «Biedermeier», war eine typische Umbruchszeit. In manchem Mitbürger waren die traditionellen Herrschafts-, Gesellschafts- und Glaubensformen, trotz zeitgenössischer Infragestellung, noch lebendig. Wolf entstammte einem alten Zürcher Geschlecht, das mit den übrigen Standesfamilien verwandtschaftlich verflochten war. Es wies eine ansehnliche Reihe namhafter Pfarrer und Gelehrter auf, doch brach die Verbindung zum ursprünglichen Handwerkerstand nie ab. Der Vater war Pfarrer in Fällanden, starb aber schon 1827, worauf die Familie nach Zürich heimkehrte.

Es fällt auf, wie stark Vorbilder Rudolf Wolf geprägt haben. In Zürich absolvierte er zuerst «den 3jährigen Curs in der Kunstschule», um Kaufmann zu werden; «nebenbey» war er «Cadett und Turner» (Tagebuch S. $1^{5}$ ). 1831 kam er ans Technische Institut, wo er «besonders die mathematischen Vorlesungen von Hr. Dr. (Karl Heinrich) Graeffe (1799-1873) besuchte», denn die Bekanntschaft mit dem Geodäten Johann Heinrich Denzler (18141876) und mit Stadtingenieur Johannes Fehr (jun., 1796-1830) liessen ihn «die Carrière eines Ingenieurs» wählen. Der Bruder Jean (1813-1839) war 


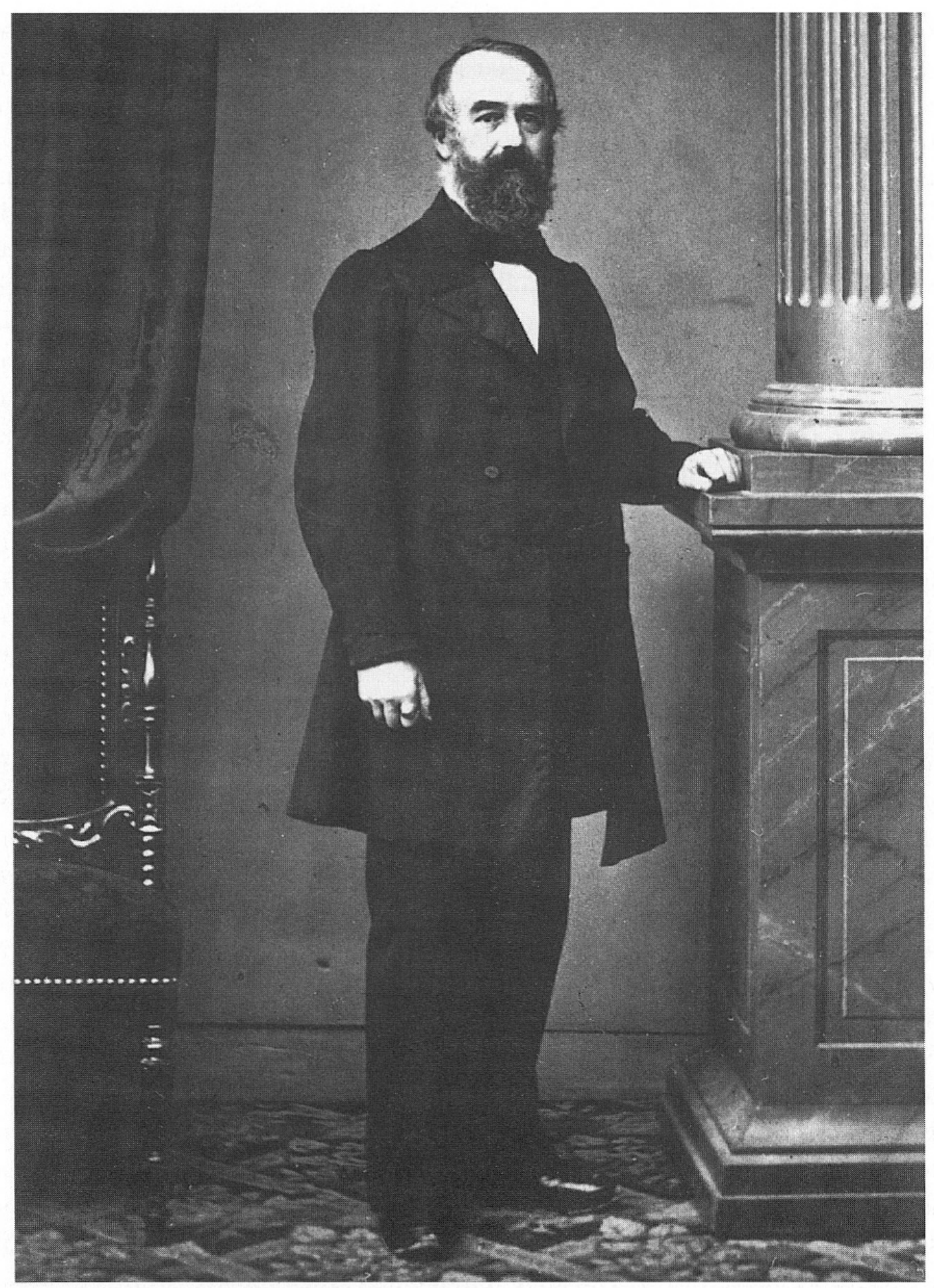

Rudolf Wolf als wohlbestallter Professor für Astronomie am Eidgenössischen Polytechnikum (heute Eidgenössische Technische Hochschule) und an der Universität Zürich (Atelieraufnahme um 1860 : Wissenschaftshistorische Sammlungen der ETH-Bibliothek) 
für die geistliche Laufbahn bestimmt. 1833 immatrikulierten sich beide an der neu errichteten Universität, der erst 17jährige Rudolf in Mathematik und Physik. Hier hatte Joseph Ludwig Raabe (1801-1859) «durch seine unermüdliche Dienstfertigkeit in Privaterklärungen grossen Einfluss auf meine Studien. Durch Herrn (Johannes) Eschmann (1808-52) erkannte ich ihre angenehme Seite ${ }^{6}$. In Hrn. Hofrath (Johann Caspar Horner, 17741834) erfüllten mich die Wissenschaften mit Ehrfurcht.» (Tb. 2). Der introvertierte Bruder übte über Rudolf «eine auf das Alter und, wie er meinte, auch auf seine tiefere Einsicht gegründete Suprematie aus ${ }^{7}$, der dieser mit der Eifersucht des Jüngeren teils durch Nachfolge, teils aber mit Opposition begegnete. Dies beispielsweise im religiösen Bereich : «Bey der Confirmation fiel mir das Gelübde sehr schwer; nicht dass ich mich gerade an bestimmten Punkten stiess, - aber die Vernunft stiess sich schon am kindlichen Glauben. Ich konnte kein bestimmtes Ja hervorbringen.» (Tb.2). Dementsprechend heisst es noch in Pfarrer Bions Rede bei der Abdankung, als «freier Geist» habe Wolf «keiner sichtbaren Konfession» angehört ${ }^{8}$. Als Staatsbürger erfüllte er zwar seine Pflicht und hatte seine eigene (eher konservative) Meinung zum Tagesgeschehen; im übrigen lag Politik dezidiert «ausser seiner Sphäre ${ }^{9} »$.

Dem Studium der Astronomie, Mathematik, Physik widmete Wolf drei Wiener, ein Berliner und 1/2 Pariser Semester. Ansporn zu eigenem Tun vermittelte ihm vor allem Joseph Johann Littrow (1781-1840) mit seinen historischen Interessen und den «Annalen der Wiener Sternwarte». In Berlin aber war es der «Pestalozzianer» Jakob Steiner (1796-1863), dessen weitgehend autodidaktischer, anekdotenreicher Werdegang ihn inspirierte. Aber auch Leben und Werk von Horners Mentor, des Freiherrn Franz Xaver von Zach (1754-1832), müssen Wolf stark beeindruckt haben, und er scheute die Mühe nicht, eigens zu der von Zach begründeten Sternwarte auf dem Seeberge bei Gotha zu pilgern. Dabei war er alles andere denn ein Eigenbrötler. Mit Vergnügen genoss er nach der Mühe des Triangulierens im Felde den Feierabend und anschliessende Ausflüge. Freundschaft, patriotische Turnund Schützenfahrt, Fest, Vergnügen, Theater, geselliges Beisammensein nahmen im Tagesplan des Studenten einen wichtigen Platz ein. Im Ausland sorgten Schweizerzirkel für heimatlichen Rückhalt. Zu Wien war er ein richtiger «Theaternarr». Darüber hinaus allerdings pflegte Wolf selten Bekanntschaften, ausser mit den Professoren und den Logisgebern. Jeans unerwarteter Tod 1839 kittete die Familie auf Mutter Regula (geb. Gossweiler, 1780-1867), die Schwester Lisette (1804-1881) und Rudolf zusammen. 
Kaum hatte dieser in Bern seine erste Stelle angetreten, folgten ihm die beiden Frauen nach. In Zürich, ab 1855, sollte Freund Wild der vierte im Familienbunde werden. Robert Billwiller, der als Assistent seinen Chef gut kannte, umriss dessen spätere persönliche Verhältnisse treffend:

\begin{abstract}
Wolfs Privatleben floss «äusserst friedlich, ruhig, einfach und regelmässig dahin». Die Gemeinschaft mit Mutter und Schwester «liess das Bedürfnis zur Gründung einer eigenen Familie nicht hervortreten, und so blieb er unverheiratet, sein Leben ganz der Arbeit weihend». Die nötige Erholung «fand er einerseits jeweils in einer gemütlichen Abendstunde unter Freunden und nähern Bekannten, anderseits bei den regelmässig im Spätsommer unternommenen Wanderungen nach ihm lieb gewordenen Orten des Vaterlandes». Dabei nahm er oft einen Schüler mit. «Wolf war in jeder Beziehung ein vortrefflicher Charakter, friedfertig, neidlos, einfach und bescheiden, ein biederer Eidgenosse. Seine ungekünstelte Herzlichkeit, sein unbegrenztes Wohlwollen, seine überall zu Tage tretende Ruhe und Herzensgüte waren nur der natürliche Ausfluss seines harmonischen und durchaus reinen Wesens.» 10
\end{abstract}

\title{
Der Lehrer
}

Bei Wolfs vielfältigen Anlagen war es nicht zuletzt der Zufall, der ihn ins Lehrfach und nach Bern lenkte - «da sich ein definitives Amt in seiner Vaterstadt für ihn nicht zeigen wollte ${ }^{11}$ ». Andere Menschen zu führen lag dem Pfarrerssohn wohl nahe. Jedenfalls vermerkte das Tagebuch minutiös die einschlägigen Tätigkeiten. Pädagogische Erfahrungen sammelte Wolf auch als Oberturner. Während des Studiums im Ausland stand die exakte Wissenschaft und ihre Geschichte im Vordergrund. Dozenten, bei denen er (manchmal allerdings nur kurz) hospitierte, erteilte der an guten und persönlichen Unterricht Gewöhnte im Tagebuch nicht ungern scharfe Zensuren. Ein Vikariat für seinen Mathematiklehrer Karl Heinrich Graeffe (1799 1873) an der Obern Industrieschule füllte den Frühling 1839. «Am Anfang war ich etwas befangen, so dass ich nicht ganz klar und geordnet sprechen konnte, - bald ging es aber besser», kommentierte er für sich den ersten Schultag (Tb. 96). Durch Vermittlung des Physikers Albert Mousson (18051890) eröffnete sich ihm eine Festanstellung - aber in Bern, als Mathematiklehrer an der Realschule, dem damals modernen Schultyp; er griff zu.

Wolf selber wünschte fürs erste ein Provisorium - «bis entschieden ist, ob diese Stelle geeignet ist, mich für meinen Lebenszweck, dem Vaterland und der Ausbreitung der exakten Wissenschaften zu nützen, arbeiten zu lassen» (Tb.98). Er unterrichtete wöchentlich in den oberen Klassen Geometrie, Physik (insbesondere Mechanik) und Vermessung bzw. geodätisches Zeich- 
nen. Wie seinerzeit Steiner verfasste er sein eigenes Lehrmittel: zuerst das Büchlein «Die Lehre von den geradlinigen Gebilden in der Ebene» (1841, $1847^{2}$ ), dann ein «Taschenbuch für Mathematik und Physik» (1852). Der «Taschen-Wolf» erlebte in der Folge, als «Taschenbuch für Mathematik, Physik, Geodäsie und Astronomie», mehrere, stets erweiterte Auflagen, und noch ein Johann Jakob Burckhardt spricht begeistert von dem praktischen Nachschlagewerk. Das 1869/72 erschienene, parallel angelegte zweibändige «Handbuch der Mathematik, Physik, Geodäsie und Astronomie» ergänzte und vertiefte die fachliche wie die historische Dimension.

Populär aber wurde Wolf bei den Schülern durch sein grosses Engagement am freizeitlich organisierten Turn- und Schwimmunterricht, an dessen Abschlussfesten und der grossen Schülerreise der «guten» Oberklässler. Um eine Hochschul-Dozentur bemühte er sich schon 1839, erhielt sie aber erst 1844, zunächst unbesoldet. 1847, nach dem Rücktritt von Professor Friedrich Trechsel (1776-1849), wurde Wolf endlich «zum besoldeten Docenten für Mathematik und Astronomie und zum Vorsteher der Sternwarte ernannt». Ein Wendepunkt kam 1852, als «die philosophische Facultät der Berner-Hochschule» ihn wegen seiner Verdienste um die Sonnenfleckenforschung mit dem Ehrendoktorat auszeichnete. Wolf erhielt, «wenn auch ohne Zulage, ein Extraordinariat für Mathematik und Physik ${ }^{12} »$, und ein bescheidener Ausbau der Sternwarte ergab sich gleichen Jahres. Er war nun in Bern nicht nur als Lehrer geschätzt, sondern auch als Wissenschafter anerkannt, so dass es ihm schwer fiel, eine Rückkehr nach Zürich aktiv zu betreiben; dies besorgte an seiner Stelle die Schwester Lisette, wie Alvin E. Jaegglis köstliche Dokumentation zeigt ${ }^{13}$. Ausführlich wog er seinem Freunde Wild gegenüber das Für und Wider ab. Es war ihm wohl klar, dass er zwar das teure alte Umfeld wie auch einen «wissenschaftlicheren» Boden zurückgewänne, aber weder den neuen Bekanntenkreis, in dem ihm wohl war, noch den Lehrerfolg mitnehmen könne. Doch als ihm Erziehungsrat Alfred Escher im April 1855 die Mathematikprofessur am Zürcher Obern Gymnasium anbot, ergriff er sie, als Sprungbrett für eine künftige akademische Karriere - und konnte sie tatsächlich schon 1861 zu deren Gunsten aufgeben.

Mit der Eröffnung der Eidgenössischen Polytechnischen Schule im Herbst 1855 nahm hier Wolfs Unterricht in Astronomie für Ingenieure und Fachlehrer, eine Drittelprofessur, seinen Anfang. Mit diesen Vorlesungen erfüllte er auch die Verpflichtungen eines universitären Extraordinariates. Die Übungen fanden zuerst im traditionellen Zürcher Sternwärtchen statt, 
das für den Anfang mehr schlecht als recht genügte. «Als dann aber die Schule sich rasch ausdehnte und zudem die Astronomie für die Ingenieurschüler zum obligatorischen Fache wurde, ging es absolut nicht mehr ${ }^{14}$ ». Die Hauptschwierigkeit für einen Neubau lag, wie so oft, im Finanziellen. Wolf wäre nicht er selber gewesen, wenn er deswegen resigniert hätte; ein privater Sponsor stiftete «die schöne Summe von 25000 Franken» und brachte damit die Sache in Bewegung ${ }^{15}$ : Der Bund berappte den Bau, der Kanton besorgte den Bauplatz, Gottfried Semper übernahm Bauplanung und Ausführung und es entstand ein architektonisches Bijou, das im nächsten Abschnitt gebührend gewürdigt wird.

In erster Linie hatte die Sternwarte der Lehre zu dienen : mit Vorlesungen und Übungen für anfänglich 60 bis 100 Studierende; Forschung kam zum Zuge, sofern Personal und Material es erlaubten, Zeit und Kraft hiefür übrig blieben. Wolf erfüllte den Lehrauftrag an ETH und Universität mit einem, meist «Elemente der Astronomie» betitelten, Kolleg und mit Übungen im Beobachten (sommers) und astronomischem Rechnen (winters). Von Zeit zu Zeit las er auch Ausgewählte Partien der höheren Astronomie, Astronomiegeschichte, Himmelsmechanik, Geographische Ortsbezeichnung, 1887/88 sogar Wahrscheinlichkeitsrechnung (Methode der kleinsten Quadrate). Begutachtung der Diplomarbeiten von Ingenieuren und Fachlehrern des Polytechnikums, von Doktorarbeiten in exakten Wissenschaften an der Universität gehörte ebenfalls zu seinen Pflichten. Bei Wolfs Abdankung 1893 würdigte der Mathematikstudent Ernst Amberg (1871-1952) den Hochschullehrer wie folgt:

\begin{abstract}
Wolfs Vorlesungen seien klar aufgebaut und ausgezeichnet ausgearbeitet gewesen. Nie habe er die Hörer «durch eine Überfülle von Stoff» erdrückt, sondern lieber Weniges gut ausgefeilt geboten. Die geschichtlichen Vorlesungen habe er gerne «durch eine Reihe charakteristischer Beispiele und Episoden» veranschaulicht, den «trockenen Gegenstand durch ein passend angebrachtes Scherzwort» belebt, gleicherweise auch manchem Studenten «die Mühe der astronomischen Beobachtungen» erleichtert. «Bei den praktischen Arbeiten hielt er darauf, die Schüler selbständig arbeiten zu lassen und sie möglichst wenig zu beeinflussen.» Nicht zuletzt sei Wolf ein wohlwollender und menschenfreundlicher Examinator gewesen ${ }^{16}$.
\end{abstract}

So urteilte ein ETH-Absolvent der Fachlehrer-Abteilung (aus der bald einmal die zukunftsträchtigen Studienrichtungen für Mathematik, Physik und Naturwissenschaften hervorgehen sollten). Wie Wolfs Unterricht bei den angehenden Bauingenieuren ankam, für die ja Astronomie Pflichtfach war, ist meines Wissens nicht überliefert. Billwiller ergänzt : «Wolf tat viel Gutes 
im Stillen und half manchem seiner Schüler über die Schwierigkeiten des Lebens hinweg. Er hatte allezeit eine offene Hand auch für ihm Fernstehende, wenn sie ihm der Hülfe würdig schienen ${ }^{17}$ ».

\section{Die Eidgenössische Sternwarte in Zürich - ein gemeinsames Werk von Rudolf Wolf und Gottfried Semper ${ }^{18}$}

Nicht bei allen Bauten hatte Gottfried Semper (1803-79) einen so kompetenten und offenbar auch begeisterungsfähigen Partner wie in der Person von Prof. Wolf beim Bau der Sternwarte. So stammt das erhaltene Bauprogramm zur Sternwarte ${ }^{19}$ zwar von der Hand Sempers, geht aber sicher auf ein gemeinsames Konzept der beiden zurück. Daraus entstanden ist sowohl ein für die damalige Zeit vorbildliches Observatorium, wie wir aus zeitgenössischen Reaktionen wissen, als auch eines der architektonisch interessantesten Bauwerke Sempers.

Gottfried Semper, der seit 1834 Architekturprofessor an der Kunstakademie Dresden gewesen war und als politischer Flüchtling in London, dank seiner aus gemeinsamer Dresdner Zeit stammenden Freundschaft mit Richard Wagner als einer der Ersten und Prominentesten 1854 zum Professor am Eidg. Polytechnikum gewählt worden war, galt natürlich als idealer Architekt für die Sternwarte. Nach seinem Projekt baute der Kanton Zürich ja auch 1861-65 das Hauptgebäude der ETH. Am 25. März 1861 kaufte der Bund vom Kanton Zürich und der Spitalpflege das notwendige Terrain am Schmelzberg in Fluntern ${ }^{20}$. Zusammen mit der mit Servituten belegten Umgebung wurde dafür gesorgt, dass die Himmelsbeobachtung von dort aus nur durch den natürlichen Horizont begrenzt wurde. Semper selber stellte das Gebäude in der «Baubeschreibung über die neue Sternwarte» von $1861^{21}$ vor :

Der eigentliche Bau «ist unsymmetrisch, weil eine Unterordnung der gestellten Erfordernisse des Programms unter die Regeln der strengen Symmetrie nur durch (stets bedenkliche) Künsteleien und Scheinarchitekturen möglich geworden wäre. Vielmehr wurde erstrebt, den innern Sonderheiten der Anlage, wie sie das Programm bedingt, auch äusserlich formale Geltung zu verschaffen und so den Charakter derselben sprechender hervortreten zu lassen». Sonach besteht der Bau aus drei an einander gefügten Raumeseinheiten:

1. Mittelbau mit dem gemeinsamen Eingange, dem Vestibulum (zugleich als Sammlungssaal dienend) und dem Observatoriumsthurm, der auch die Treppe enthält.

2. westliches Nebengebäude, das zurückliegt, aber die gleiche Richtung wie der Mittelbau mit der Längenaxe von Süd nach Nord befolgt. Es enthält im Parterre das Auditorium und zwei Zimmer für den Direktor und einen Assistenten. 


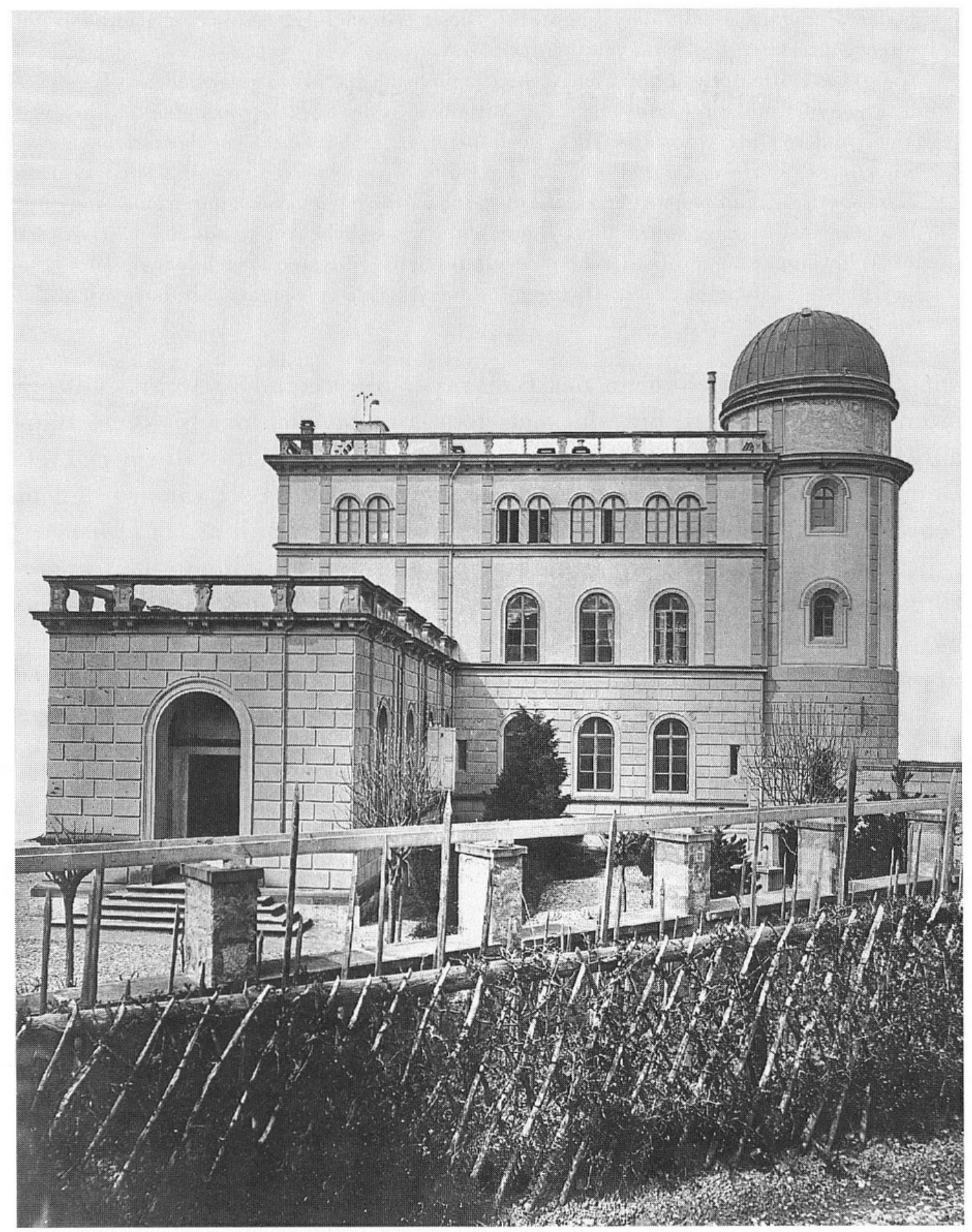

Sternwarte von Osten (Aufnahme vor 1900 : Semper-Archiv des Instituts für Geschichte und Theorie der Architektur, ETH Zürich) 
3. der Meridiansaal mit der Bibliothek. Dieser Flügel folgt mit seiner Längsaxe der entgegengesetzten Richtung von Ost nach West.

Wie in ihren Richtungen und Lagen, eben so ungleich sind diese verschiedenen Elemente des Gebäudes in ihren Elevationen. Der Mittelbau ist der höchste, wird aber nördlich von dem (runden) Observatoriumsthurm noch überragt. Er hat über dem Parterre noch zwei Stockwerke, welche die Wohnräume für den Direktor und Assistenten enthalten. Das Dach ist flach mit Asphalt bedeckt und steht für Beobachtungen im Freien durch eine Thüre mit dem Observatorium in Verbindung. Dann folgt der westliche Anbau mit einem Stockwerk für Wohnräume und darüber Dachbodengelasse. Den niedrigsten Theil bildet der Meridiansaal, dessen Bestimmung keine Überbauung zulässt. Er ist gleichfalls mit flachem Pultdache (Asphalt) bedeckt.»

Die rationalen Beziehungen der Baukörper untereinander weisen auf ein interessantes Bauwerk hin, das sich gerade nicht nach der für diese Bauaufgabe damals üblichen axialsymmetrischen Organisationsform richtet. Damit erntete Semper allerdings zu Beginn nicht nur Lob, wie aus seinem Schreiben an Schulratspräsident Dr. Kappeler (vom 3. Juni 1861) hervorgeht. Hatte doch der das Geschäft führende Bundesrat Pioda den jungen Heinrich Wild (1833-1902), Professor für Mathematik und Physik zu Bern, um ein Gutachten gebeten. Dieser erhob darin eine Reihe von Einwänden, so: ein Keller fehle, Auditorium und «Hausflur» seien zu gross, erstes Stockwerk und Turm zu hoch, die Treppe im Turm ungewohnt, die Küche dem Observatorium zu nahe. Semper, offenbar recht verärgert, nahm dazu folgendermassen Stellung:

\footnotetext{
Allgemein falle auf, dass der Gutachter die architektonischen Motive nicht beachte, ja mitunter «absichtliches Nichtsehenwollen» derselben verrate; so «p.e. bei dem Einwurfe gegen die Anlage des Vestibüls, wofür ich das Zimmer für Aufbewahrung und Aufstellung von Instrumenten vergessen haben soll. Es ist unmöglich, dass Herr P. Wild meine grossen Schränke, die gleichsam das ganze Vestibüle ausmachen, übersehen haben kann. Er müsste denn gar nichts von Plänen verstehen ...»
}

Semper betonte, dass er bei seinem Entwurfe «zunächst nur die Aufgabe des Programms im Auge hatte und dass derselbe in allen innern Theilen überlegt ist, dass er aus Motiven hervorgeht, deren Richtigkeit bestritten werden darf, die man aber ganz zu ignorieren nicht das Recht hat.»

So frage sich beim Observatorium, ob dasselbe von der Wohnung «des Direktors möglichst fern zu halten sei oder nicht? Die Gründe, welche für vollständige Isolierung sprechen, lassen sich hören, aber dann muss man nicht, wie Herr Wild es thut, die Räume, woselbst die turbulente Jugend bei Tag verkehrt, um den Fuss des Thurmes ordnen. Entweder wird nur bei Nacht beobachtet, dann stört die Nähe der Direktionswohnung nicht mehr als ein leeres Auditorium - oder es wird auch bei Tage beobachtet, dann stören 40 bis 50 Hörer und 
sonstige verkehrende Studenten in den untern Räumen des Thurmes die Beobachtungen sicher mehr, als die Nachbarschaft der Familie.

Ich habe den Thurm nicht mehr isoliert, als zu seiner Benützung nöthig schien, und ihn mit der Direktorswohnung in Verbindung gesetzt, theils aus lokalen Gründen, theils - und besonders weil ich es für wesentlich halte - damit dem Beobachter das Beobachten so bequem wie möglich gemacht werde, also aus praktisch-psychologischen Rücksichten». Das Vestibül diene «als gemeinsamer Vorplatz für das Auditorium, den Meridiansaal, das Arbeitszimmer des Direktors und dasjenige für den Assistenten (bzw. Wetterbeobachtungszimmer). Auch führt es zum Observatorium. Es verbindet also alle Theile und ist zugleich ein Museum für die Geschichte der Astronomie und Erdkunde, zugleich auch als Conservatorium der Instrumente und sonstigen astronomischen Apparate.

Dies das Motiv des Architekten. Er beabsichtigte die Förderung und Intensivierung des Studiums für die Studierenden» - zugleich aber «eine architektonische Wirkung, was ihm erlaubt sein darf, unter Vermeidung jener langweiligen obligaten Gänge, auf welche der Laie verfällt, wenn es ihm beliebt, Pläne zu machen».

Die Küche aber sei «derjenige Raum, der in einer Wohnung die regelmässigste Temperatur behält, deren Lage in der Nähe der Warte auch wohl in Fällen während der nächtlichen Operationen auf dem Observatorium bequem erscheinen dürfte. Geniert die Feuerstelle an der angegebenen Stelle, so lässt sie sich leicht weiter entfernen ...»

Der zur vollen Zufriedenheit der Auftraggeber errichtete Bau ist mit allen Zeichen der damals modernen und richtungweisenden Architektur geschmückt: Im Bewusstsein, dass Architektur polychrom zu sein hat, entwarf Semper 1863 für den Tambour des obersten Turmgeschosses ein rundumlaufendes Sgrafitto in Originalgrösse, das damals auch ausgeführt wurde - es ist allerdings längst zerstört. Semper bemühte sich auch, dem «italienischen Landsitz» (einem Bautypus, der aus den burgartigen Rittersitzen der Toscana entstanden ist) eine entsprechende Umgebung zu gestalten, und liess auf den Terrassen, wo verschiedene Forschungsaufgaben verrichtet werden konnten, Pergolen errichten, die Schatten spendeten und die Bodenreliefs unterstrichen.

Alles in allem ist aus der Zusammenarbeit der beiden Professoren ein «Gesamtkunstwerk»entstanden, das der Gründerepoche der ETH Zürich zur hohen Ehre gereicht.

\section{Forschungsorganisator und Wissenschaftshistoriker}

Wolfs erste Bekanntschaft mit «Wissenschaft», jedenfalls der büchernen, reicht ins Pfarrhaus Fällanden zurück, wo die «grosse und werthvolle Bibliothek» des Predigers, Religionslehrers und Dichters Salomon Wolf (17521810) «sorgfältig besorgt und nach Kräften vermehrt wurde»-um aber 1827 
«auf Rath sog. guter und einsichtiger Freunde in alle Welt zerstreut zu werden», wie Wolf ironisch schrieb ${ }^{22}$. In den wissenschaftlichen Alltag und Verbund führte ihn, neben dem Studium, vor allem der Geodät Eschmann ein. Dieser gründete im März 1834 «die topographische Gesellschaft, die sich vornahm, die Umgebungen Zürichs zu vermessen»; er «war Präsident, ich Aktuar und Quästor, thätiges Mitglied war noch Wild» (Tb.2). Eschmann engagierte Wolf in der Folge wiederholt zu Vermessungsarbeiten für die Dufourkarte. Der Auslandsaufenthalt von 1836-1838 brachte auch insofern Gewinn, als Wolf bedeutende Persönlichkeiten kennenlernte, deren einige ihm zeitlebens verbunden blieben. Von Gauss übernahm er die Anregung, in Zürich ein «magnetisches Observatorium» einzurichten - um «Zürichs Wissenschaftlichkeit mehr Ruf zu gewinnen» (Tb.89, 142). Er trug sie der Physikalischen Gesellschaft vor, präsidierte deren Arbeitsgruppe, verfocht sie auch vor der Schweizerischen Naturforschenden Gesellschaft - letztlich ohne konkretes Ergebnis, da niemand «die Oberleitung übernehmen wollte» (Tb.124). Erfolg hatte ein zweiter, weniger kostspieliger Antrag: die Physikalische Gesellschaft Zürich ernannte noch 1839 Steiner und Littrow zu ihren Ehrenmitgliedern (Tb. 128).

In Bern trat Wolf gleich nach Stellenantritt in die Naturforschende Gesellschaft ein, die von der Umtriebsamkeit des quirligen Zürchers bald profitierte. 1841 wurde er Sekretär (-1854) und Kassier (-1845), 1843 Redaktor ihrer neuen «Mittheilungen» (-1855). Diese dienten ihm auch für seine eigenen Veröffentlichungen. Hier verdiente er sich seine Sporen als Bibliothekar ab (1841-1847), indem er Bibliothek und Archiv (samt Autographensammlung) neu ordnete, organisierte und - öfters beschenkte. 1855 betraute der Berner Verein ihn mit dem Präsidium; zeitlebens blieb er Mitglied. Es war wohl fast selbstverständlich, dass er in Zürich die Redaktion der neuen «Vierteljahrsschrift der Naturforschenden Gesellschaft» (1856 ff.) übernahm - und sich damit hier die gleichen publikatorischen Annehmlichkeiten eröffnete; dieses Amt nahm ihm erst der Tod.

Bezeichnenderweise wurde Wolf zum Bibliothekar des Polytechnikums gewählt. Hier, wie auch auf der Sternwarte, konnte sich sein Sammler- und Schenkertrieb tummeln - sofern ihm Zeit dazu blieb. «Die Bibliothek des Polytechnikums», so schrieb er 1880 in der von ihm zum 25jährigen Schuljubiläum bestellten Festschrift, hatte in erster Linie «den mathematischen Wissenschaften und den technischen Fächern zu dienen». Wolf erfüllte diese Aufgabe pflichtschuldig, mit zuerst einem, später zwei Gehilfen, bescheidenen Krediten und Gebühren und einer gekonnten Geschenkpolitik, so dass 
der Nachfolger Prof. Ferdinand Rudio 1894 immerhin eine Bücherei von rund 36000 Bänden vorfand. Wolf begründete ein recht praktisches, grobsystematisches Standortnummern-System und veröffentlichte insgesamt fünf Kataloge, den letzten 1876, sein Supplement 1887. Paul Scherrer, der vierte Nachfolger im Bibliothekariat, zollte Wolfs Akzessionspolitik hohe Anerkennung:

\begin{abstract}
«Trotz der Notwendigkeit, mit sehr begrenzten Mitteln aus dem Nichts aufzubauen, vermied er, die Neugründung in naheliegender Beschränkung auf Utilität und Aktualität zu einer der damals aufkommenden «Special Libraries` mit rasch veraltenden Beständen zu machen. Er gab ihr schon im Frühstadium die breite Bildungsgrundlage einer Hochschulbibliothek, die den Zusammenhang des Werdenden mit dem Gewesenen festhält. Sein weitreichendes Interesse für die Geschichte der Wissenschaften verschaffte ihr neben der momentgebundenen Gebrauchsliteratur die kostbaren Original- und Frühausgaben von Werken zur Astronomie, Geodäsie, Mathematik, Mechanik und Physik aus dem 16. bis 18. Jahrhundert, wie auch eine wertvolle Sammlung alter Militaria, die Bibliothek der Mathematisch-militärischen Gesellschaft Zürich. Ihm gebührt das entscheidende Verdienst, der Bibliothek damit über das Fachliche hinaus den dauerhaften kulturellen Rang gesichert zu haben ${ }^{23} . »$
\end{abstract}

Wenn heute die Wissenschaftshistorischen Sammlungen der ETH-Bibliothek über einen guten Fundus naturwissenschaftlicher Originalausgaben des 16.-18. Jahrhunderts verfügen, so ist dies gutenteils das Verdienst Wolfs. Dessen Sammeltätigkeit beschränkte sich aber keineswegs auf Bücher. Die stark biographische Ausrichtung brachte es mit sich, dass er schon früh sich auch um Bildnisse einschlägiger Persönlichkeiten kümmerte. Bald fügten sich astronomische und mathematische Instrumente dazu, die er zu einer kleinen musealen Sammlung in der Eingangshalle der Zürcher Sternwarte vereinigte und in einem Sammlungsverzeichnis minutiös beschrieb.

Schweizer Wissenschaftsgeschichte zu betreiben, stand Wolf, wie er dem Tagebuch 1839 anvertraute, seit Studienbeginn, «obschon noch nie so hell wie jetzt, vor der Seele» (Tb. 115). An der Jahresversammlung der Schweizerischen Naturforschenden Gesellschaft 1841 in Zürich stellte er einen Publikationsplan vor. Er beabsichtige, eine «Specialgeschichte der Naturlehre und ihrer Hülfswissenschaften» zu verfassen, und zwar eine «Schweizergeschichte», die zusammen mit andern Länder-Monographien ermögliche, «die Geschichte der exacten Wissenschaften und ihrer Anwendungen» zu schreiben ${ }^{24}$. Die Vielfalt seiner Berner «Zusatzbeschäftigungen» liess den Plan nicht allzuweit fortschreiten. Die ersten Zürcher Jahre verschafften ihm offenbar etwas mehr Musse; vier Bände «Biographien zur Kulturgeschichte der Schweiz» (1858-1862) waren die Frucht davon. Wolf verstand 
es, sie «durch reichlich eingestreute Briefauszüge zu ungemein lebensvollen Bildern zu gestalten ${ }^{25}$ ). Während geschichtlich interessierte Naturwissenschafter das Werk auch heute noch seines Detailreichtums wegen schätzen, ist es sonst samt seiner Fortsetzung, den «Notizen zur schweizerischen Kulturgeschichte ${ }^{26}$ », kaum noch bekannt.

Seine wesentlichen Beiträge zur modernen Wissenschaftsorganisation hat Wolf zumeist selber am besten beschrieben, vor allem in der «Geschichte der Vermessungen in der Schweiz» (1879) - an deren einem Hauptresultat, der Dufourkarte, er in jungen Jahren ja mitgewirkt hatte. Es sind dies: die Neuorganisation der Sternwarten zu Bern und Zürich, die Begründung der Sonnenflecken-Statistik, der Schweizer Beitrag zur internationalen Gradmessung und schliesslich die Meteorologische Zentralanstalt.

Die (seit 1812 auf der «grossen Schanze» nordwestlich der Stadt bestehende) bernische Sternwarte übernahm Wolf 1847; das «von mächtigen Bäumen umschattete Häuschen» sah aber «eher wie die Grabstätte der Urania als wie eine unter ihren Schutz gestellte Werkstätte aus, und ich muss mich jetzt fast verwundern, dass ich damals den Mut fand den Versuch zu wagen, dem verkümmerten Institute bessere Tage zu verschaffen, - aber es gelang nach und nach meinem festen Willen ...» : Die Bäume wurden gefällt, «1848 ein Vorzimmerchen und ein Kabinetchen mit Kamin» angebaut, und «so konnte ich doch immerhin, ausser annähernden Zeitbestimmungen und meteorologischen Aufzeichnungen, einige werthvolle Beobachtungsserien über Sonnenflecken, Sternschnuppen, das Alpenglühen \&c. beginnen ${ }^{27}$ ». Obschon Wolf auf der Bildungsreise kaum eine der Sternwarten, die auf seinem Wege lagen, ausgelassen hatte, unternahm er 1847, «um dem neuen Amte möglichst gewachsen zu sein», nochmals eine Sternwartentour nach Bonn, Hamburg, Altona, Berlin, Leipzig und München ${ }^{28}$. Nach 1852 unterstützte Wolfs internationales Renommee den weiteren Fortschritt. Auch Zürichs Sternwarten haben eine ähnliche anekdotenreiche Geschichte. Das erste kleine und mühevolle Observatorium befand sich im sog. Karlsturm des Grossmünsters, wo sich der unglückselige Pfarrer Heinrich Waser (174280) seiner bediente. Dank Horner und Stadtingenieur Johannes Feer sen. (1763-1823) wurde 1810 bei der Kronenpforte ein «astronomisches Hüttchen» gebaut und für Ortsbestimmungen, Beobachtungen und Unterricht benützt ${ }^{29}$, so noch von Wolf selber. Dank seinem Einsatz konnte es 1864 durch die Sempersche Sternwarte ersetzt werden. Über die hier neben der Lehrverpflichtung ausgeführten wissenschaftlichen Arbeiten orientierten Wolfs «Astronomische Mittheilungen». 
Eng mit der Einrichtung der Berner und Zürcher Sternwarten verknüpft sind die Bemühungen um die Sonnenflecken-Forschung. Wolf begann mit der Bestandesaufnahme Ende 1847 und führte sie in der Folge solange er lebte weiter. 1843 hatte Gottfried Schwabe zu Dessau die Periodizität der Sonnenflecken entdeckt; fast gleichzeitig mit Edward Sabine und Alfred Gautier fiel Wolf die Parallelität mit dem Erdmagnetismus auf. Für eine genaue Ermittlung des Periodizitätszyklus führte er die sog. Relativzahl ein. Historische Studien liessen ihn «die Epochen der Maxima und Minima bis zum Beginn des 17. Jahrhunderts bestimmen und daraus mittlere Periodenlängen von 11,11 Jahren ableiten. Wolfs Nachforschungen führten nach und nach zu dem Erfolg, dass heute zuverlässige Monatsmittel der Relativzahlen seit 1749 bekannt sind und seit 1849 lückenlose Tageswerte ${ }^{30} . »$ Um die Ausfälle der bedeckten Tage auszugleichen, organisierte Wolf sich einen freiwilligen Mitarbeiterstab. Seit 1928 koordiniert die Internationale Astronomische Union IAU auch diese Tätigkeit.

1861 lancierte der preussische Geodät General Baeyer den Plan einer europäischen Gradmessung, um die Abweichungen der Erde vom geometrischen Ellipsoid zu ermitteln. Die Schweizerische Naturforschende Gesellschaft befürwortete das Forschungsvorhaben für die Schweiz und bestimmte eine eigene Kommission zu seiner «ernstlichen Förderung» - mit Wolf als Präsidenten, General Dufour, den Professoren Elie Ritter, Adolphe Hirsch und Heinrich Denzler als Beisitzern. Baeyer, Wolf und seine Kommission einigten sich auf ein Detailprogramm, die Gesellschaft validierte es an ihrer Luzerner Jahresversammlung 1862, Wolf verfasste eine Erläuterungsbroschüre für die Mitglieder der Bundesversammlung, diese bewilligte den Kredit, und 1863 trat die Schweiz der Unternehmung offiziell bei. Ausgeführt wurden in der Folge astronomische Arbeiten (Ermittlung der PolarKoordinaten der Schweizer Sternwarten, u.a.m.), an denen Wolf sich persönlich beteiligte, trigonometrische Nach- und Neumessungen sowie ein «Präzisions-Nivellement». Die Unternehmung wurde aber «nach allen Dimensionen und Anforderungen» ausgedehnt und erforderte daher ein Mehrfaches an Zeit und Geld als vorgesehen ${ }^{31}$. Die «Arbeiten der geodätischen Commission» legen davon Zeugnis ab. Wolf behielt das Präsidium bis 1893 .

Schon zur Zeit der Aufklärung, Helvetik und Restauration bemühten sich Schweizer Gelehrte und Gesellschaften, ein meteorologisches Beobachtungsnetz aufzubauen. Auch Wolf erhob zu seiner Berner Zeit meteorologische Daten und publizierte sie, getreu dem Wahrspruch des Arztes und Astronomen Olbers, dass nur Veröffentlichung sie sichere. 1860 nahm die 
Schweizerische Naturforschende Gesellschaft einen neuen Anlauf und setzte erst eine vorberatende, 1862 eine ausführende Kommission ein; ihr gehörte auch Wolf an. Bundesversammlung und «eine Reihe von Kantonen» sprachen die nötigen Kredite, worauf die Messinstrumente bestellt, das Stationennetz aufgebaut und eine Datensammelstelle errichtet wurde: die von Wolf betreute und ebenfalls in der Sternwarte domizilierte Meteorologische Centralanstalt. Ein Assistent und «ein bis zwei Hülfsrechner» standen ihm zur Seite ${ }^{32}$. Alljährlich gab er «in einem stattlichen Bande die Resultate der schweizerischen Beobachtungsstationen heraus, lieferte selbst häufig wissenschaftliche Beiträge» und publizierte meteorologische Geschichtsquellen ${ }^{33}$. Als die Aufgaben wuchsen und 1881 die Meteorologische Centralanstalt zum Bundesinstitut erhoben wurde, gab Wolf seine Leitungsfunktion ab; Assistent Dr. Robert Billwiller (1845-1905) wurde ihr erster Direktor.

Wolfs fachhistorische Beschlagenheit, die eng mit seinem Ruf als Solarforscher verknüpft war, blieb nicht verborgen. Die Historische Commission der kgl. Akademie der Wissenschaften München beauftragte ihn, im Rahmen ihrer «Geschichte der Wissenschaften in Deutschland» den Band über die Astronomie zu schreiben. Dabei war ihm sein sprachenkundiger Meteorologieassistent Billwiller «bei Sammlung des Materials durch vielfache Auszüge und Übersetzung» behilflich ${ }^{34}$; das Werk erschien 1877. Schon zwei Jahre später konnte Wolf seine «Geschichte der Vermessungen in der Schweiz als Historische Einleitung zu den Arbeiten der schweiz. geodätischen Commission» veröffentlichen. Wolfs letztes grosses Opus war das «Handbuch der Astronomie, ihrer Geschichte und Litteratur» (1890-1893, in vier Halbbänden). Es war konzipiert sowohl als einführendes Kompendium für Studierende als auch bequemes «Nachschlagebuch» für die Fachgenossen, die hier «auf einem gedrängten Raume eine Menge von sachlichen und historisch-litterarischen Angaben aller Art vereinigt finden, welche sie sonst aus Hunderten von Bänden zusammensuchen müssten ${ }^{35}$ ). Der kundige Billwiller kommentiert dieses, wie Wolfs historiographisches Schaffen überhaupt, folgendermassen:

Wolfs Schlusswerk «war eine Umarbeitung seines Handbuchs der Mathematik, Physik, Geodäsie und Astronomie (1869/72)», welches «seinerseits die detaillierte Entwicklung» des «Taschen-Wolf» $\left(1852^{1}, 1877^{5}\right)$ gegeben hatte. Es enthalte die Frucht langjährigen Studiums «und eines erstaunlichen Sammelfleisses». Der Leser finde darin «höchst bedeutsame Resultate eigener Forschungen», z. B. «über ältere astronomische Instrumente, über die Entwicklung der Trigonometrie und die Erfindung der Logarithmen» (Jost Bürgi).

Zwar treffe zu, «dass Wolf sich in seinen historischen Studien auf die Ermittlung der Thatsachen beschränkt hat und dass eine philosophische Vertiefung in den Gegenstand 
nicht seine Sache war. So mag man wohl in seiner Geschichte der Astronomie bei der Darstellung und Behandlung der Probleme von allgemeinem Interesse den kulturhistorischen Hintergrund vermissen. Allein Wolfs Natur, die schlichte, einfache, jeden Prunk meidende Art seines Wesens prägt sich auch in seinen litterarischen Produkten aus. Er beschränkte sich in seinen, in knapper, präciser Form gegebenen Darstellungen immer ganz auf die Sache selbst, teilte das Tatsächliche möglichst vollständig mit, hielt zwar oft mit der Kritik nicht zurück, vermied aber Reflexionen, die über den Kreis des behandelten Gegenstandes hinausreichten. In Anbetracht, dass Wolfs Bildungsgang nicht auf humanistischer Basis ruhte, dass er die Kenntnis der alten Sprachen, die sich bei seinen historischen Studien sehr oft als notwendig erwiesen hatten, sich also, soweit möglich, mühsam erwarb, wird man seine eminenten historisch-litterarischen Leistungen eher bewundern müssen als bemängeln dürfen. Wie seine schriftlichen Arbeiten, so war auch sein mündlicher Vortrag beim Unterricht knapp, aber gründlich und klar, ohne das Beiwerk von Phrasen» ${ }^{36}$. 


\section{Anmerkungen und Literaturangaben}

1 Ausser Dr. Fröhlich (vgl. Anm. 18) habe ich meinem Kollegen Michael Beckett zu danken für die englische Zusammenfassung.

2 In Umschrift vervielfältigt : Schriftenreihe der ETH-Bibliothek, 30 (erscheint voraussichtlich Ende 1993). Zu Wolfs Leben und Werk vgl. auch: H. Lutstorf, Professor Rudolf Wolf und seine Zeit (Schriftenreihe der ETH-Bibliothek, 31); ferner das Wolfs Andenken gewidmete Dezemberheft 1993 der Vierteljahrsschrift der Naturforschenden Gesellschaft Zürich.

3 Joseph Scherrer: Johann Wolf; ein schweiz. Studirender in seinem Bildungsgange. 1840.

4 Verzeichnet in: Handschriften und Autographen der ETH-Bibliothek, Nr.6; vgl. auch Nrn. 116, 120, 121.

5 Weitere Tagebuch-Zitate sind im Text analog (Tb.x) vermerkt.

6 Vgl. hierzu Wolfs Geschichte der Vermessungen, S.254 u.a.a.O. sowie Wolfs von J.J. Burckhardt in: Die Alpen. Zeitschrift des SAC, 65 (1989) S. 90-101 publizierte Beschreibung einer Triangulationsreise mit Eschmann.

7 Scherrer 1.c., S. 137.

8 Reden gehalten bei der Trauerfeierlichkeit für Herrn Dr. J. Rudolf Wolf ... Zürich 1894. S. 6 .

9 Wolf an Georg von Wyss, 7.5.1845 (Familienarchiv von Wyss, Zentralbibliothek Zürich).

10 Billwiller in seinem Nachruf auf Wolf: Verhandlungen der Schweiz. Naturforschenden Gesellschaft 1894, S.247-8.

11 Billwiller, S.239.

12 R. Wolf, Geschichte der Vermessungen in der Schweiz, 1879: S. 285-6.

13 Alvin E. Jaeggli, Die Berufung des Astronomen Joh. Rudolf Wolf nach Zürich 1855 (Schriftenreihe der ETH-Bibliothek, 11; Zürich 1968).

14 Wolf, Das Schweizerische Polytechnikum. Historische Skizze zur Feier des 25jährigen Jubiläums 1880. S. 27.

15 Wolf, Geschichte der Vermessungen, S.299.

16 Reden gehalten bei der Trauerfeierlichkeit für Herrn Dr. J. Rudolf Wolf ... Zürich 1894, S. 14-15.

17 Billwiller in Wolfs Nekrolog, 1.c. S. 248.

18 Beschrieben von Dr. Martin Fröhlich, Denkmalpfleger der bundeseigenen Bauten im Amt für Bundesbauten, Bern.

19 Im Semper-Archiv des Instituts für Geschichte und Theorie der Architektur (gta) der Eidg. Technischen Hochschule Zürich.

20 Ausgefertigt von der Staatskanzlei, signiert vom Staatsschreiber Gottfried Keller.

21 Siehe Anmerkung 19.

22 Neujahrsblatt Zürich: Waisenhaus, 1874. S.21 Anm.95.

23 P. Scherrer, «Bibliothek»; in: Festschrift ETH 1855-1955, S. 528.

24 Verhandlungen der Schweiz. Naturforschenden Gesellschaft 26 (1841), S. 203-9.

25 A. Wolfer, Nekrolog auf Wolf: Vierteljahrsschrift der Astronomischen Gesellschaft 29 (1894), S. 7.

26 Einer Rubrik der Vierteljahrsschrift der Naturforschenden Gesellschaft Zürich; sie hielt sich bis 1942. 
27 Geschichte der Vermessungen, S. 285-6.

28 W. Oechsli in seiner ETH-Festschrift 1905, S. 199.

29 Wolf, Geschichte der Vermessungen, S. 218.

30 M. Waldmeier, 100 Jahre Sonnenfleckenstatistik. Astronomische Mitteilungen der Eidg. Sternwarte Zürich, Nr. 152 S.6.

31 Wolf, Geschichte der Vermessungen, S. $304 \mathrm{ff}$.

32 Wolf, Geschichte der Vermessungen, S. 302.

33 Wolfer S.9. Ab 1861 erschienen die Schweiz. meteorologischen Beobachtungen, hg. v.d. Meteorologischen Centralanstalt Zürich (ab 1880: Annalen der Schweiz. meteorol. Centralanstalt); ab $1880 \mathrm{ihr}$ laufender Wetterbericht. Fürs Ältere s. a. Supplementband (I) der Annalen.

34 Vorwort S. VII. Reprints : 1933, 1965.

35 Vorwort im 1. Halbband Seite V. Reprints : 1973.

36 Billwiller 1.c., S. 246-7.

Dr. Beat Glaus

ETH-Bibliothek

CH-8092 Zürich 